\title{
Clinical Application Prospect of Human Synovial Tissue Stem Cells from Osteoarthritis Grade IV Patients in Cartilage Regeneration
}

\author{
Rizki Rahmadian ${ }^{1,2 *}$, Marlina Adly ${ }^{3}$, Ismail Hadisoebroto Dilogo ${ }^{4}$, Gusti Revilla ${ }^{5}$ \\ ${ }^{1}$ Doctoral Program, Faculty of Medicine, Andalas University, Padang, Indonesia; ${ }^{2}$ Department of Orthopedics and Traumatology, \\ Faculty of Medicine, Andalas University, M.Djamil General Hospital, Padang, Indonesia; ${ }^{3}$ Faculty of Farmacy, Andalas \\ University, Padang, Indonesia; ${ }^{4}$ Department of Orthopaedics and Traumatology, Faculty of Medicine, Indonesia University, Dr. \\ Cipto Mangunkusumo General Hospital, Jakarta, Indonesia; ${ }^{5}$ Department of Anatomy, Faculty of Medicine, Andalas University, \\ Padang, Indonesia
}

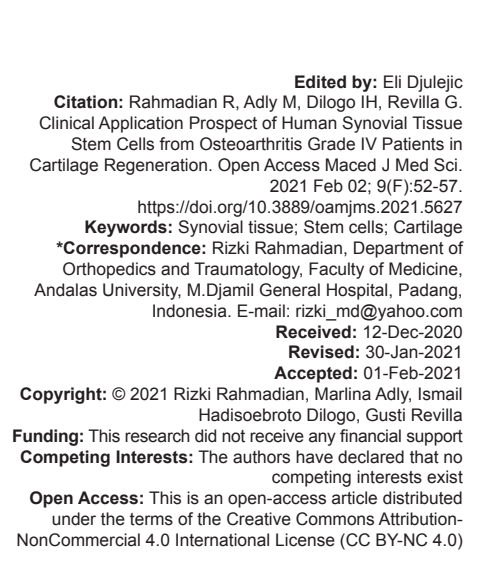

Abstract

Osteoarthritis $(\mathrm{OA})$ is a joint problem that continues to increase in prevalence as life expectancy increases. OA can affect any joint, especially those that support body weight such as the knee and hip joint. Although both primary and secondary OA have the same clinical symptoms, it can be caused by different etiologies. OA is no longer considered a degenerative disease, although age is still a major factor. Various attempts have been made to regenerate joint cartilage damaged by OA. The use of stem cells in OA therapy is a very promising opportunity. Stem cells are undifferentiated biological cells and are multipotent to differentiate into specific cells. In principle, local stem cells are the best source of stem cells to regenerate the surrounding tissue. The synovial membrane is a tissue in the joint that can regenerate. After synovectomy surgery, repair, and growth of synovial tissue occur rapidly. Synovial tissue as a source of stem cells only provides a limited amount. One source of synovial tissue that can be used is tissue taken from the total knee replacement process in grade 4 OA patients. However, it is necessary to prove the potential of synovial tissue stem cells originating from old-age donors.

\section{Introduction}

Osteoarthritis $(O A)$ is a joint degenerative disease that affects many people throughout the world. $\mathrm{OA}$ is a very complex disease and can affect all joint components, especially joint cartilage and subchondral bone [1]. The main pathophysiology of OA occurs in cells and the extracellular matrix. The process of joint damage due to $\mathrm{OA}$ involves all joint components including joint cartilage, subchondral bone, ligament, meniscus, joint capsule, and synovial tissue. The complexity of the pathophysiology that occurs in OA makes no single therapy able to resolve the problem completely. So that the development of stem cells therapy is one solution to this problem [2]. Moreover, the use of stem cells originating from the synovial membrane (SM) is one of the best alternative sources [3].

The cause of $\mathrm{OA}$ is multifactorial so that until now the cause of $\mathrm{OA}$ is not known with certainty. In terms of etiology, OA is divided into two causes, primary and secondary OA. Primary OA occurs due to age, weight, and activity. Whereas secondary $\mathrm{OA}$ is caused by other diseases that affect the joints and can trigger the $\mathrm{OA}$ process to occurfaster such as: Joint infections, rheumatoid arthritis, gout, joint dysplasia, malalignment, and posttraumatic injuries that cause injury to parts of the joint such as anterior cruciate ligament rupture, torn meniscus, and intraarticular fractures. Abnormal development of joints, calcium crystal deposition, neuropathy, hemophilia, metabolic disorders (rickettsia, hemochromatosis, chondrocalcinosis, and ochronosis), and endocrine abnormalities (acromegaly, hyperparathyroidism, and hyperuricemia) can also cause secondary OA [4], [5], [6].

OA occurs because of interactions between systemic factors and local factors. Systemic factors include age, sex, genetics, hormones, and diet. Meanwhile, the local factors that play a role in the OA process are joint injury, obesity, and activity [7].

\section{Pathogenesis}

The main damage in OA occurs in joint cartilage, although in principle all joint components 
also experience changes. Network changes in the OA process are the result of a combination of mechanical, cellular, and biochemical interactions [8]. Chondrocytes as the main constituent of joint cartilage have receptors to respond to biomechanical disorders around the cartilage, such as growth factors, cytokines, and other inflammatory mediators. In the OA process, there is an imbalance between pro-inflammatory cytokines that degrade the matrix, with anti-inflammatory cytokines and growth factors that prevent catabolic molecules.

Matrix metalloproteinase (MMP) is an enzyme that plays a role in the degradation of the matrix in OA. This enzyme is divided into five groups based on the specific substrate, namely collagenase, stromelysin, gelatinase, membrane-type MMPs (MT-MMP), and other types. MT-MMP has been identified in bone tissue. MT1-MMP can initiate the activation of proMMP-2 and proMMP-13, where both enzymes can mediate damage to type II collagen in cartilage. Based on this information, it can be seen that MMP is responsible for cartilage destruction in OA [9], [10], [11]. Collagenases such as MMP-1, MMP-8, and MMP-13 can only degrade the helical domain of type II collagen, while MMP-3 degrades collagen types IX and XI [9]. MMP-13 plays a dominant role in the progression of cartilage degeneration with the main target being collagen [12].

Other enzymes that play a role to degrade the cartilage matrix are disintegrin and metalloproteinase with thrombospondin motifs (ADAMTS). Aggrecanase has the most central role with MMP to degrade the matrix as ADAMTS-4 and ADAMTS-5. ADAMTS-4 is active during cartilage degeneration and its expression is increased in degenerative cartilage. ADAMTS-5 is present in normal cartilage and OA conditions [12].

Other mediators in an anabolic activity besides anti-inflammatory cytokines and growth factors are the tissue inhibitors of metalloproteinase (TIMP), which include the main inhibitors of tissue. TIMP is produced by fibroblasts and chondrocytes. Genomically, there are four types of TIMP, namely TIMP-1, TIMP-2, TIMP3 , and TIMP-4. TIMP-1 is a protective mediator and appears to be exceptionally high in $\mathrm{OA}$ in synovial fluid. TIMP-1 and TIMP-2 are both present in the SM of OA [13], [14], [15]. The expression of mRNA from TIMP by chondrocytes in OA cartilage is higher than TIMP by normal cartilage chondrocytes. MMP activity can be inhibited by TIMP in tissue destruction, although not significantly. A balance between MMP and TIMP activity is needed to maintain cartilage homeostasis. Research found increased MMP expression in early OA, both in humans and experimental animals. These results strongly indicate that OA therapy design is needed to specifically inhibit MMP [9].

The pathophysiological process in OA joints is also the result of mediating pro-inflammatory cytokines and enzymes that degrade the matrix in catabolic activity [16]. Complex molecular mechanisms that overlap with each other produce tissue damage to the joints [13]. The molecular mechanism of OA is explained through the involvement of cytokines, chemokines, and inflammatory mediators in chondrocyte metabolism, which includes anabolism and catabolism activity [17], [18]. Unbalanced chondrocyte metabolism is caused by the overproduction of pro-inflammatory cytokines and enzymes that play a role in degrading the matrix. This is related to the decreased anabolic signaling, resulting in the destruction of the extracellular matrix and continued to cartilage degradation. The disruption of cartilage homeostasis is also caused by the presence of oxidative stress that results from reactive oxygen species (ROS). Furthermore, the process of catabolism will occur through cell apoptosis induction, destruction of matrix components, increased production of enzymes that will degrade the matrix and inhibit matrix synthesis, and oxidation of intracellular and extracellular molecules. An increase in catabolism activity and decreased anabolism activity cause a disturbed balance of extracellular matrix and consequently cartilage degradation. An imbalance between anabolic and catabolic activity at the molecular level results in OA, where the primary injury response occurs in joint cartilage [19], [20]. The overall condition of the joint depends on the expression of adequate growth factors, such as increased synthesis of transforming growth factor $\beta$ (TGF- $\beta$ ) and insulin-like growth factor (IGF) in the extracellular matrix [12], [13].

In the process of OA, interleukin-1 (IL-1) and tumor necrosis factor- $\alpha$ (TNF- $\alpha$ ) lead to chemotaxis and leukocyte degranulation. These cytokines also increase the expression of inflammatory mediators and the activity of several proteolytic enzymes such as MMPs and aggrecanase that play a role in degrading joint cartilage [10], [13], [20], [21], [22]. Molecules included in pro-inflammatory cytokines include IL-1 $\beta$ (IL-1 $\beta$ ), TNF$\alpha, \mathrm{IL}-6, \mathrm{IL}-15, \mathrm{IL}-17$, and IL-18 [7], [13], [14], [16], [23] (Figure 1).

$\mathrm{IL}-1 \beta$ is one of the most influential cytokines in the pathogenesis of OA and is widely produced by synovial and chondrocyte macrophages in the OA joint [14]. These cytokines can induce inflammatory reactions and catabolic effects independently [16]. The role of $\mathrm{IL}-1 \beta$ in pathogenesis is to suppress the production of type II collagen and proteoglycans and stimulate synovial cells to release MMPs [14]. IL-1 $\beta$ cytokines also release proteoglycans from the extracellular matrix into the synovial fluid. Besides, IL-1 $\beta$ can inhibit the synthesis of collagen types II, IX, and $\mathrm{XI}$, as well as stimulate the production of abnormal proteoglycans, and decrease TIMP expression [13]. As for its role in stimulating, the production of ROS, such as peroxide and hydroxylate radicals that can damage joint cartilage directly [16]. 


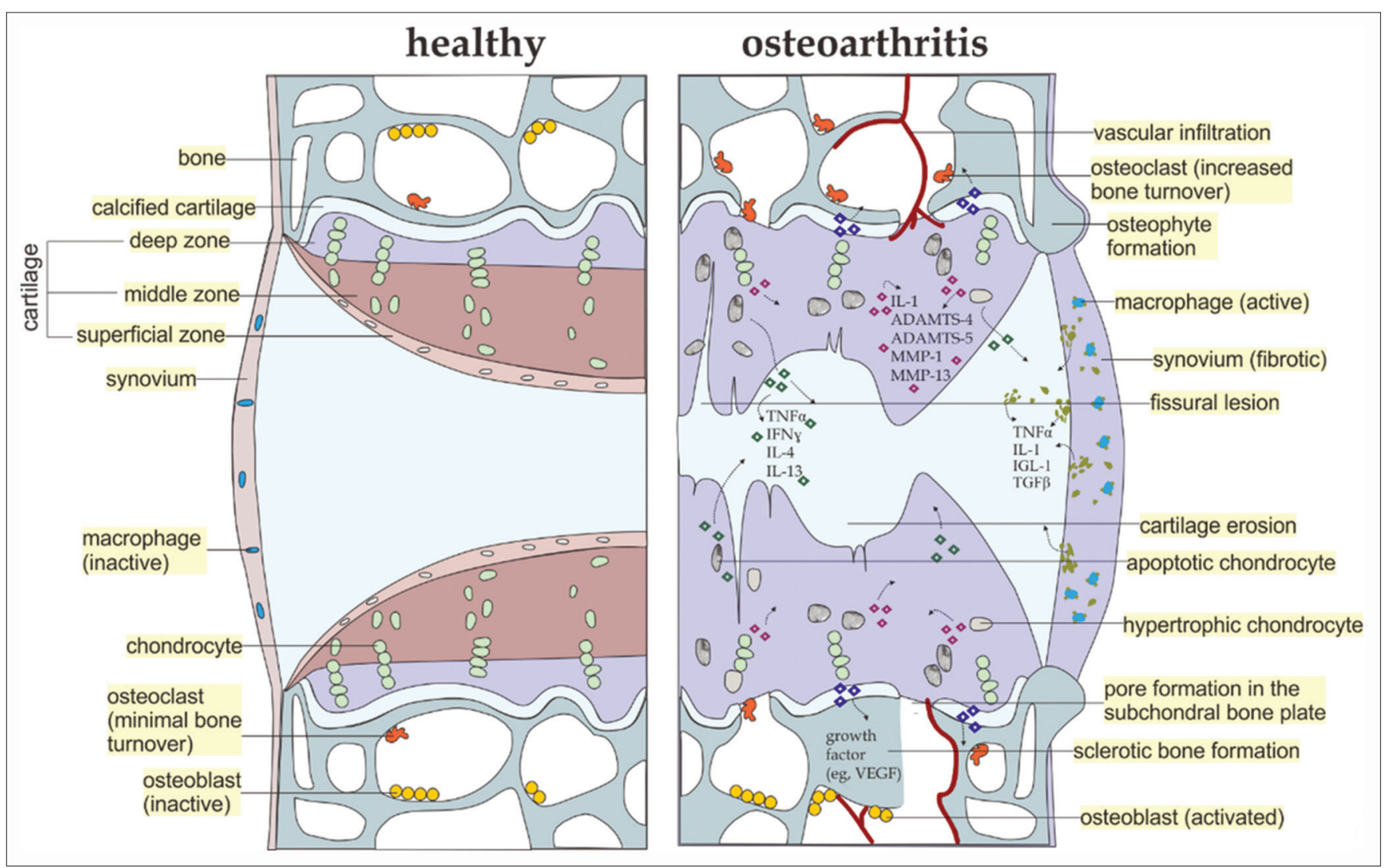

Figure 1: Signaling pathway and structural changes in osteoarthritis joint cartilage

Besides IL-1 $1 \beta$, TNF- $\alpha$ is an important inflammatory cytokine associated with the pathophysiological process when OA occurs. There is a synergy between the two cytokines of the many phenomena of OA. In the OA disease process, TNF- $\alpha$ affects the blockade of chondrocyte synthesis from proteoglycan components, proteoglycan protein binding, and type II collagen [16].

\section{Stem Cells Therapy for OA}

OA is caused by a complex pathophysiologic mechanism involving cytokines and inflammatory mediators, and as of to date no single drug has been able to overcome the inflammatory mediators involved. Various attempts of therapy are used to regenerate the tissue around the knee joint, especially cartilage joints, to reduce the complaints caused by OA and help the knee joint to function optimally. Current developing treatments are derived from a cellular product such as platelet-rich plasma, bone marrow aspirate, and mesenchymal stem cells (MSC) [24].

Stem cells possess a therapeutic effect through differentiation ability, immunity regulation, antiinflammatory, improving microenvironmental conditions, and enhancing the regeneration process [25]. MSC have multipotent progenitor capabilities derived from adult stem cell (ASC) populations that can be found in some tissues such as bone marrow, peripheral blood, adipose tissue, synovium tissue and fluid, placenta, and umbilical cord. A cell qualifies as an MSC if it meets the requirements set by the International Society for Cellular Therapy. The terms of conditions are having the ability to stick on the surface of the cup when cultured in a plastic adherent cup and having a cluster of differentiation proteins surface molecules, namely CD73, CD90, and CD105. Unlike hematopoietic stem cells, MSC do not express CD34, CD14, CD45, and human leukocyte antigen-DR. Furthermore, they can differentiate through three main pathways of mesenchymal, that is: Osteogenic (becoming bone cells/osteocytes), chondrogenic (becoming cartilage cells), and adipogenic (becoming fat cells/ adipocytes) [26]. With its chondrogenic potentials, MSC and their immunosuppressive characteristics become the major modalities in the treatment of OA.

In the process of differentiation chondrogenic culture, MSC are strengthened by the role of several growth factors such as IGF-1, fibroblast growth factor 2 (FGF-2), epidermal growth factor (EGF), and plateletderived growth factor (PDGF). Moreover, the potential of chondrogenic culture can also be increased by the addition of non-protein components such as dexamethasone and ascorbic acid. The oxygen tension factor is one of the factors that influence the occurrence of chondrogenic differentiation. Low oxygen levels can 
trigger chondrogenic differentiation while Col2A1 and SOX9 gene expression also increase proteoglycan synthesis and type II collagen [27]. Physical stimulation can also affect calcium channels and the chondrogenic process. With an increase in concentration, intracellular calcium can initiate a signaling pathway which can increase the expression of a cartilage-specific gene and produce protein-specific cartilage during the differentiation process of MSC [28].

MSC are a multipotent type of ASC. Adult MSC can be isolated from several tissues such as bone marrow, adipose tissue, placenta, umbilical cord blood, periosteum, and other tissues that are the source of stem cells closest to damage. Compared to other sources, MSC derived from bone marrow and synovium have greater chondrogenic potential [29].

MSC have the immunosuppressive ability by lowering $\mathrm{T}$ cell proliferation through the secretion of pro-inflammatory cytokines. These cells also produce inflammatory mediators such as prostaglandins E2 and IL-6. Together with TGF- $\beta$, MSC improve the chondrogenesis process. Previous studies found that several growth factors, such as TGF- $\beta$, IGF-1, bone morphogenic protein (BMP), FGF, and EGF were used in in vitro cartilage tissue engineering to direct chondrogenic phenotypes, stimulate extracellular matrix production, and direct chondrogenesis [30], [31], [32].

In studies related to differentiation, adult MSC experienced changes that occur in the cellular microenvironment. Genes that do not show expression in specialized proresolving mediator (SPM) will appear when SPM differentiates into chondrocytes. These genes include glypican-3, cadherin-11, MMP-7 or type II collagen. On the contrary, other genes such as CX3CR1, MMP-1, and MMP-9 decreased the expression [33].

\section{Possibility of Synovial Tissue Stem Cells from OA Patient Grade IV}

SM can regenerate very well. This can be seen from the rapid growth of synovial tissue after synovectomy. This regeneration speed shows that the SM has the potential to repair damaged tissue and has potential as a source of stem cells. In different circumstances, the ability of synovial chondromatosis is a rare proliferative condition and the etiology is unknown. This raises the possible role of SM-MSC in producing several intrasynovial cartilaginous nodules. MSC isolated from the SM called SM-MSC or synovial mesenchymal progenitor cells, are known to have the same phenotype and function as MSC. The presence of MSC in the synovial layer raises questions about its origin. One of the possible origins of the presence of
MSC in the synovial layer is from blood that penetrates the synovial tissue or comes from bone marrow that is connected to the intraarticular space.

Local MSC in the joint can be found in almost all of the diarthrodial joint tissues, as shown in Figure 2. SM-MSC has the same characteristics as other MSC sources, which can regenerate through chondrogenic, osteogenic, and adipogenic processes, and in certain circumstances have the ability of myogenesis. These stem cells are also found to have heterogeneous clonal abilities when applied to other types of populations. The studies that transplanted SMSC in mice found regeneration ability in anterior tibial muscle injury. When compared with other stem cell sources, those originating from tissues around the joints obtained SMSC with the highest chondrogenic ability [34].

The role of MSC in the synovial layer is related to the healing potential of tissue originating from the mesoderm. Furthermore, cells in the synovial layer are involved in the early osteoarticular disease. Bone marrow-MSC (BM-MSC) and SM-MSC express different genes, including activin $A$, which are regulated in BM-MSC. Unlike BM-MCS, SM-MSC derived from the SM maintains a proliferation rate and the potential for the formation of SM-MSC which has a high selfrenewal ability and multipotentiality inherent in a single cell. Single cells originating from SM-MSC become heterogeneous during the expression process. Nonclonal plastic adherent synovial MSCs consist of a uniform set of cells. Koga et al. showed that synoviumderived MSCs transplanted in rabbits were converted into microenvironments [3].

Cells that originate from the human SM are known to have the best chondrogenesis potential among other mesenchymal cells and one of the sources that may be representative to repair damaged cartilage. One study revealed that SM-MSC which was cultured with human autologous serum could differentiate into chondrocytes in vitro. However, the potential for estrogen is lower compared to cells implanted in fetal serum bovine. Synovial cells originating from older patients with OA can be reprogrammed into pluripotent cells in alginate culture by stimulating BMP-2 or BMP-7 in dexamethasone and non-serum conditions. The results show that the SM has the therapeutic potential for the treatment of joint cartilage damage using in vitro experiments because the human autologous serum is known to increase the proliferative potential of SM-MSC through activating the PDGF signaling. Cells that resemble SM-MSC can be found in healthy cartilage or cartilage that has OA [3].

Bone marrow cells that are embedded or present in growth factors such as TGF- $\beta$, BMP, and IGFs, have an important role in repairing cartilage damage. BMP especially BMP-7 and IGF-1 have shown the ability in vitro to stimulate chondrogenesis. The problem will remain in the complexity of the signaling pathways involved in chondrogenesis, which are 


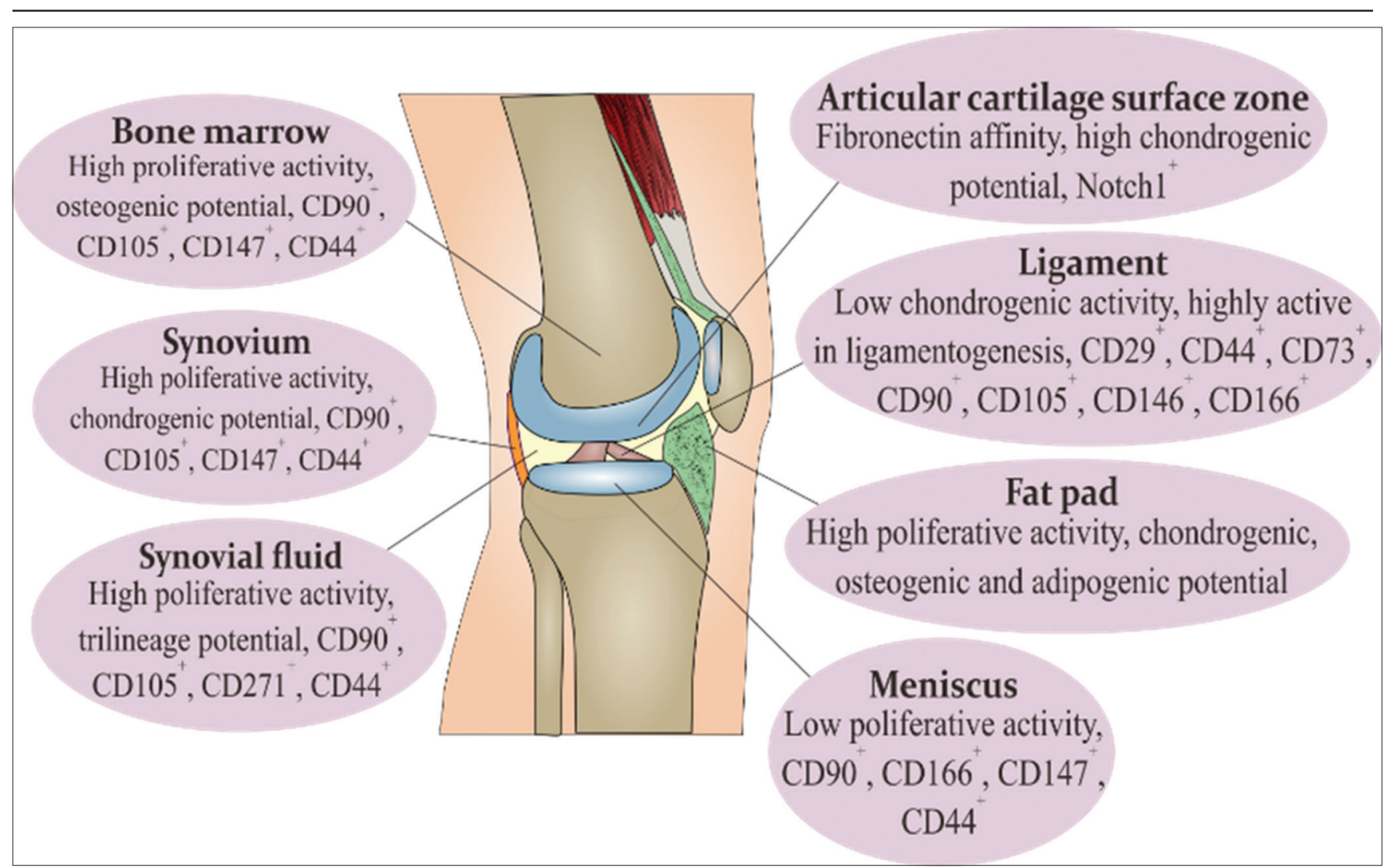

Figure 2: Characterization of stem cell surface markers of various tissues in the diarthrodial joint

stimulated by cell-to-cell contact. The profile of healthy and arthritis synovium fluid chemokines can contribute to the recruitment of human mesenchymal progenitors from bone subchondral tissue. Synovial fluid in healthy humans, patients with $\mathrm{OA}$, and rheumatoid arthritis contain different levels of chemokines such as CCL22, CCl27, CXCL5, and CXCL12 which can inhibit the migration of human subchondral mesenchymal progenitors. Other chemokines that can be found in synovium fluid such as CCL2, CCL24, and CXCL7 do not affect mesenchymal progenitor cells. The number of SPM recruited by synovial fluid from patients with rheumatoid arthritis is lower than that of OA or normal humans. This shows that chemotactic factors contribute to the attractiveness of the progenitor. There are different aspects of cell morphology that come from healthy human synovium fluid and those suffering from OA. This comparison shows that the SPM-MS level in the normal knee joint increases 7 times during the initial phase of OA. SPM-MS may play a role in homeostasis, remodeling, and tissue repair through cell turnover [3].

\section{Conclusions}

MSC derived from the SM of the knee joint in patients with grade VI OA have the potential to regenerate joint cartilage.

\section{References}

1. Indonesian Rheumatology Association. Diagnosis dan Penatalaksanaan Osteoarthritis; 2014. p. 2-3.

2. Kolf CM, Cho E, Tuan RS. Mesenchymal stromal cells. Biology of adult mesenchymal stem cells: Regulation of niche, selfrenewal and differentiation. Arthritis Res Ther. 2007;9(1):204.

PMid:17316462

3. de Sousa EB, Casado PL, Neto VM, Duarte ME, Aguiar DP. Synovial fluid and synovial membrane mesenchymal stem cells: Latest discoveries and therapeutic perspectives. Stem Cell Res Ther. 2014;5(5):112. https://doi.org/10.1186/scrt501 PMid:25688673

4. Kuszel L, Trzeciak T, Richter M, Czarny-Ratajczak M Osteoarthritis and telomere shortening. J Appl Genet. 2015;56(2):169-76. https://doi.org/10.1007/s13353-014-0251-8 PMid:25366419

5. Michael JW, Schlüter-Brust KU, Eysel P. The epidemiology, etiology, diagnosis, and treatment of osteoarthritis of the knee. Dtsch Arztebl Int. 2010;107(9):152-62. https://doi.org/10.3238/ arztebl.2010.0152 PMid:20305774

6. Sinkov V, Cymet T. Osteoarthritis: Understanding the pathophysiology, genetics, and treatments. J Natl Med Assoc. 2003;95(6):475-82.

PMid:12856913

7. Ashkavand $Z$, Malekinejad $H$, Vishwanath BS. The pathophysiology of osteoarthritis. J Pharm Res. 2013;7(1):132-8.

8. Dewing K, Setter SM, Slusher BA. Osteoarthritis and Rheumatoid Arthritis 2012: Pathophysiology, Diagnosis, and Treatment, Nurse Practitioner Healthcare Foundation; 2012.

9. Ishiguro N, Kojima T, Poole AR. Mechanism of cartilage 
destruction in osteoarthritis. Nagoya J Med Sci. 2002;65(3-4):73-84.

PMid:12580533

10. Loeser R. Molecular mechanisms of cartilage destruction in osteoarthritis. J Musculoskelet Neuronal Interact. 2008;8(4):303-6.

PMid:19147949

11. Tchetina EV. Developmental mechanisms in articular cartilage degradation in osteoarthritis. Arthritis. 2011;2011:683970. https://doi.org/10.1155/2011/683970

PMid:22046522

12. Wang M, Shen J, Jin H, Im HJ, Sandy J, Chen D. Recent progress in understanding molecular mechanisms of cartilage degeneration during osteoarthritis. Ann N Y Acad Sci. 2011;1240:61-9. https:// doi.org/10.1111/j.1749-6632.2011.06258.x

PMid:22172041

13. Carmona JU, Prades M. Pathophysiology of osteoarthritis. Compend Equine. 2009;4(1):28-40.

14. Monemdjou R, Kapoor M. Synovium in the pathophysiology of osteoarthritis. Clin Pract. 2010;7(6):661.

15. Sellam J, Berenbaum F. The role of synovitis in pathophysiology and clinical symptoms of osteoarthritis. Nat Rev Rheumatol. 2010;6(11):625-35. https://doi.org/10.1038/nrrheum.2010.159 PMid:20924410

16. Wojdasiewicz P, Poniatowski ŁA, Szukiewicz, D. The role of inflammatory and anti-inflammatory cytokines in the pathogenesis of osteoarthritis. Mediators Inflamm. 2014;2014:561459. https://doi.org/10.1155/2014/561459 PMid:24876674

17. Goldring MB, Otero M. Inflammation in osteoarthritis. Curr Opin Rheumatol. 2011;23(5):471-8. PMid:21788902

18. Madry H, Luyten FP, Facchini A. Biological aspects of early osteoarthritis. Knee Surg Sports Traumatol Arthrosc. 2012;20(3):407-22. https://doi.org/10.1007/s00167-011-1705-8 PMid:22009557

19. Ahmed S, Wang N, Hafeez BB, Cheruvu VK, Haqqi TM. Punica granatum L. extract inhibits IL-1beta-induced expression of matrix metalloproteinases by inhibiting the activation of MAP kinases and NF-kappaB in human chondrocytes in vitro. J Nutr. 2005;135(9):2096-102. https://doi.org/10.1093/jn/135.9.2096 PMid: 16140882

20. Burnett BP, Levy R, Cole BJ. Metabolic mechanisms in the pathogenesis of osteoarthritis-a review. J Knee Surg. 2006;19(3):191-7. PMid:16893158

21. Grenier S, Bhargava MM, Torzilli PA. An in vitro model for the pathological degradation of articular cartilage in osteoarthritis. J Biomech. 2014;47(3):645-52. https://doi.org/10.1016/j. jbiomech.2013.11.050 PMid:24360770

22. Sokolove J, Lepus CM. Role of inflammation in the pathogenesis of osteoarthritis: Latest findings and interpretations. Ther Adv Musculoskelet Dis. 2013;5(2):77-94. https://doi. org/10.1177/1759720x12467868

PMid:23641259

23. Glyn-Jones S, Palmer AJ, Agricola R, Price AJ, Vincent TL,
Weinans $\mathrm{H}$, et al. Osteoarthritis. Lancet. 2015;386(9991):37687. https://doi.org/10.1016/s0140-6736(14)60802-3 PMid:25748615

24. Harrison-Brown M, Scholes C, Hafsi K, Marenah M, Li J, Hassan $F$, et al. Efficacy and safety of culture-expanded, mesenchymal stem/stromal cells for the treatment of knee osteoarthritis: A systematic review protocol. J Orthop Surg Res. 2019;14(1):34. https://doi.org/10.1186/s13018-019-1070-8 PMid:30683159

25. Song Y, Zhang J, Xu H, Lin Z, Chang H, Liu W, et al. Mesenchymal stem cells in knee osteoarthritis treatment: A systematic review and meta-analysis. J Orthop Translat. 2020;24:121-30.

PMid:32913710

26. Kim C, Keating A. Cell therapy for knee osteoarthritis: Mesenchymal stromal cells. Gerontology. 2019;65(3):294-8. https://doi.org/10.1159/000496605

PMid:30897578

27. Harrell CR, Markovic BS, Fellabaum C, Arsenijevic A, Volarevic V. Mesenchymal stem cell-based therapy of osteoarthritis: Current knowledge and future perspectives. Biomed Pharmacother. 2019;109:2318-26. https://doi. org/10.1016/j.biopha.2018.11.099

PMid:30551490

28. Uzieliene I, Bernotas P, Mobasheri A, Bernotiene E. The role of physical stimuli on calcium channels in chondrogenic differentiation of mesenchymal stem cells. Int J Mol Sci. 2018;19(10):2998. https://doi.org/10.3390/ijms19102998 PMid:30275359

29. Emadedin M, Aghdami N, Taghiyar L, Fazeli R, Moghadasali R, Jahangir $\mathrm{S}$, et al. Intra-articular injection of autologous mesenchymal stem cells in six patients with knee osteoarthritis. Arch Iran Med. 2012;15(7):422-8. https://doi.org/10.1016/j. jcyt.2014.01.035 PMid:22724879

30. Kock L, van Donkelaar CC, Ito $K$. Tissue engineering of functional articular cartilage: The current status. Cell Tissue Res. 2012;347(3):613-27. https://doi.org/10.1007/ s00441-011-1243-1 PMid:22030892

31. Lam J, Lu S, Kasper FK, Mikos AG. Strategies for controlled delivery of biologics for cartilage repair. Adv Drug Deliv Rev. 2015;84:123-34. https://doi.org/10.1016/j.addr.2014.06.006 PMid:24993610

32. Zhao C, Tan A, Pastorin G, Ho HK. Nanomaterial scaffolds for stem cell proliferation and differentiation in tissue engineering. Biotechnol Adv. 2013;31(5):654-68. https://doi.org/10.1016/j. biotechadv.2012.08.001

PMid:22902273

33. Demoor M, Ollitrault D, Gomez-Leduc T, Bouyoucef $M$, Hervieu M, Fabre H, et al. Cartilage tissue engineering: Molecular control of chondrocyte differentiation for proper cartilage matrix reconstruction. Biochim Biophys Acta. 2014;1840(8):2414-40. https://doi.org/10.1016/j.bbagen.2014.02.030 PMid:24608030

34. Barry F, Murphy M. Mesenchymal stem cells in joint disease and repair. Nat Rev Rheumatol. 2013;9(10):584-94. https://doi. org/10.1038/nrrheum.2013.109

PMid:23881068 